\title{
Two Simple Rules for Properly Formatting Numbers
}

\author{
Mark B. Mycyk
}

Published online: 31 January 2012

(C) American College of Medical Toxicology 2012

Keywords Scientific writing · Numbers · Grammar · Manuscript

Most submissions to the Journal of Medical Toxicology and other scientific journals include numbers from a dataset somewhere in the manuscript. Knowing when to spell out a number distinguishes a seasoned writer from a new one.

RULE 1 If you begin a sentence with a number, spell out the number.

\section{Example 1}

Incorrect: 67 subjects in the cohort reported hair loss after exposure to thallium.

Correct: Sixty-seven subjects in the cohort reported hair loss after exposure to thallium.

M. B. Mycyk $(\square)$

Toxikon Consortium, Cook County Hospital,

1900 West Polk Street, 10th Floor,

Chicago, IL 60612, USA

e-mail: mycyk.mark@gmail.com
RULE 2 Do not mix a spelled out number with a symbol or unit: spell out the word for the symbol or unit.

\section{Example 2}

Incorrect: Twenty-three $\%$ of the lead-poisoned patients in our randomized clinical trial requested another course of chelation therapy.

Correct: Twenty-three percent of the lead-poisoned patients in our randomized clinical trial requested another course of chelation therapy.

A number that appears anywhere else in a sentence does not need to be spelled out. If you are unhappy with the appearance of a sentence on the page because it starts with a spelled out number, reorder your sentence so that number no longer starts it.

Example 1 (reordered): Hair loss was reported by 67 subjects in the cohort after exposure to thallium.

Example 2 (reordered): Of all the lead-poisoned patients in our randomized clinical trial, $23 \%$ requested another course of chelation therapy.

Remembering these two simple rules when including numbers in your next submission to $J M T$ will give your manuscript the polish of a seasoned writer. 